\title{
Integrated Registration, Segmentation, and Interpolation for 3D/4D Sparse Data
}

\author{
Adeline Paiement \\ Department of Computer Science, Merchant Venturers Building, Woodland Road, Bristol BS8 1UB, UK \\ Thesis Advisors: Prof. Majid Mirmehdi \\ Date and location of PhD thesis defense: $12^{\text {nd }}$ February 2014, University of Bristol, Bristol, UK
}

Received 24th February 2015; accepted 14th May 2015

\begin{abstract}
We address the problem of object modelling from 3D and 4D sparse data acquired as different sequences which are misaligned with respect to each other. Such data may result from various imaging modalities and can therefore present very diverse spatial configurations and appearances. We focus on medical tomographic data, made up of sets of 2D slices having arbitrary positions and orientations, and which may have different gains and contrasts even within the same dataset, as illustrated by the cine cardiac MRI in Fig. 1 and the multi-modal brain MRI in Fig. 2. The analysis of such tomographic data is essential for establishing a diagnosis or planning surgery.
\end{abstract}

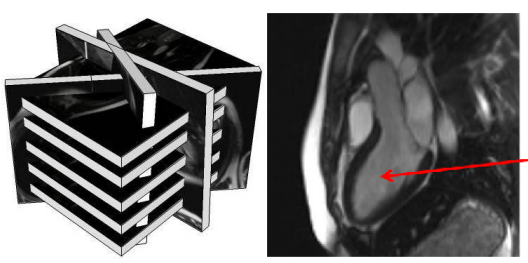

(a)

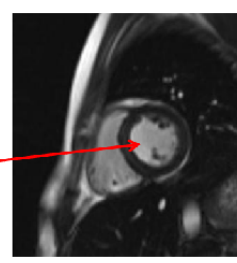

(b)

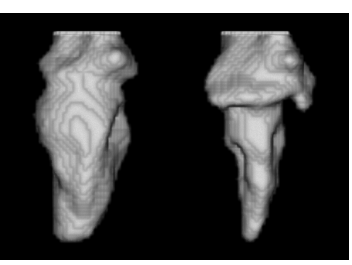

(c)

Figure 1: Cardiac cine MRI: A typical example of tomographic data having varying spatial configurations, gaps, and intensities. (a) spatial configuration of slices, (b) one vertical (long-axis) and one horizontal (shortaxis) slice both showing the same region of the heart, (c) and (d) output 4D model of the left ventricle of the heart seen at two timeframes.

Modelling from sparse and misaligned data requires solving the three inherently related problems of registration, segmentation, and interpolation. We propose a new method to integrate these stages in a level set framework. Registration is particularly challenging by the limited number of intersections present in a sparse dataset, and interpolation has to handle images that may have very different appearances [1]. Hence, registration and interpolation exploit segmentation information, rather than pixel intensities, for increased robustness

Correspondence to: <csatmp@bristol.ac.uk>

Recommended for acceptance by Jorge Bernal

DOI http://dx.doi.org/10.5565/rev/elcvia.712

ELCVIA ISSN:1577-5097

Published by Computer Vision Center / Universitat Autònoma de Barcelona, Barcelona, Spain 


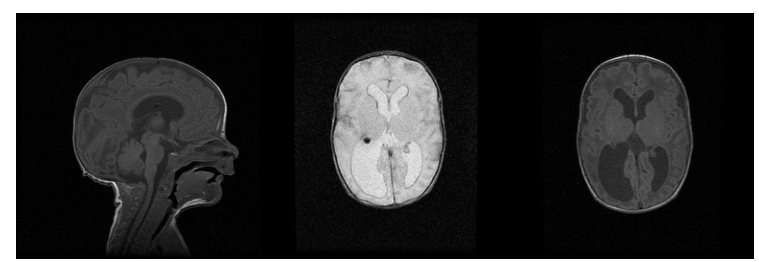

(a) (b)

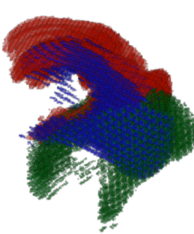

(d)

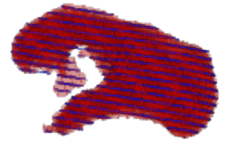

(e)

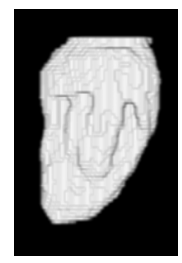

(f)

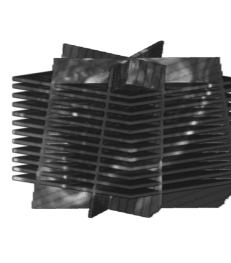

(g)

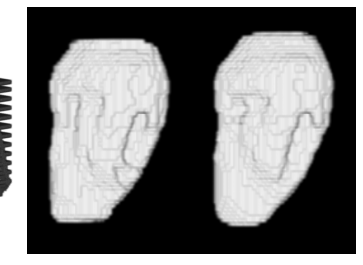

(h)

(i)

Figure 2: Comparison of the proposed and sequential approaches. Top row: Comparison of registration when modelling the brain ventricles from multi-modality MRIs - (a) to (c) example images from each of the three sets of slices of the dataset, (d) and (e) registration results of the sequential and proposed approaches, respectively. Bottom row: Comparison of interpolation when modelling the left ventricle of the heart from an artificial cine cardiac MRI - (f) ground-truth shape used to produce the artificial dataset, (g) spatial configuration of the dataset, with gaps between the slices requiring interpolation, (h) and (i) modelling results from the sequential and proposed methods, respectively.

and accuracy. We achieve this by first introducing a new level set scheme based on the interpolation of the level set function by radial basis functions [2]. This new scheme can inherently handle sparse data, and is more numerically stable and robust to noise than the classical level set. We also present a new registration algorithm based on the level set method, which is robust to local minima and can handle sparse data that have only a limited number of intersections. Then, we integrate these two methods into the same level set framework [3].

The proposed method is validated quantitatively and subjectively on artificial data and MRI and CT scans. It is compared against a state-of-the-art, sequential method comprising traditional mutual information based registration, image interpolation, and 3D or 4D segmentation of the registered and interpolated volume. In our experiments, the proposed framework yields similar segmentation results to the sequential approach, but provides a more robust and accurate registration and interpolation. In particular, the registration is more robust to limited intersections in the data and to local minima, as shown in the top row of Fig. 2. The interpolation is more satisfactory in cases of large gaps, due to the method taking into account the global shape of the object, and it recovers better topologies at the extremities of the shapes where the objects disappear from the image slices. As a result, the complete integrated framework provides more satisfactory shape reconstructions than the sequential approach.

\section{References}

[1] Adeline Paiement, Majid Mirmehdi, Xianghua Xie, Mark Hamilton, Simultaneous Level Set interpolation and segmentation of short- and long-axis MRI. Proceedings of Medical Image Understanding and Analysis (MIUA) 2010, pp. 267-272. July 2010.

[2] Adeline Paiement, Majid Mirmehdi, Xianghua Xie, Mark Hamilton, Integrated Segmentation and Interpolation of Sparse Data. IEEE Transactions on Image Processing, Vol. 23, Issue 1, pp. 110-125, 2014. 
[3] Adeline Paiement, Majid Mirmehdi, Xianghua Xie, Mark Hamilton, Registration and Modeling from Spaced and Misaligned Image Volumes. Submitted to Medical Image Analysis. 